Plant Tissue Cult. \& Biotech. 28(1): 35-44, 2018 (June)

$\overline{\mathrm{PTC} \& \mathrm{~B}}$

\title{
Over Expression of $p 5 c s$ Gene to Increase Cold Stress Tolerance in Iranian Species of Petunia
}

\section{Maryam Jamshidnia *1, Cyrus Ghobadi, Badraldin Ebrahim Sayed Tabatabaei and Ahad Yamchi ${ }^{2}$}

Department of Agricultural Biotechnology, College of Agriculture, Isfahan University of Technology, Isfahan 84156-83111, Iran.

Key words: Over expression, $p 5 c s$ gene, Cold stress, Petunia sp.

\begin{abstract}
In order to examine the relationship between proline and cold stress in Iranian (local) genotype of petunia, p5cs gene was transferred to petunia through Agrobacterium-mediated transformation. The leaf discs from four weeks and shoot apices from 7-day-old petunia in vitro plants were co-cultivated with Agrobacterium strain LBA4404 harboring a plasmid pBI121 as the vector system for transformation of petunia. pBI121 plasmid containing $\beta$-glucuronidase (gus) gene as a reporter gene and $\Delta 1$-pyrroline-5-carboxylate synthetase ( $p 5 c s)$ gene and neomycin phosphotransferase (nptII) gene were used as a selectable marker. The co-cultivated leaf discs and shoot apices were transferred to the selective medium and thereafter to proliferation medium respectively. To confirm transformation, regenerated plants were subjected to the polymerase chain reaction (PCR), GUS histochemical and proline assays. The results confirmed the presence of the gus and $p 5 c s$ gene in the genome of all transformants and the transformed plants were more tolerant $(674.87 \mu \mathrm{g} / \mathrm{g}$ fresh leaves) than the wild types under stress conditions.
\end{abstract}

\section{Introduction}

Plants are most often experienced environmental stresses, such as drought, salinity, low and high temperatures. In order to survive, plants activate different mechanisms to sense and respond to the stresses. Proline as an important osmolite has a major role in reducing osmotic pressure of the cell which is under stresses such as low temperature, lack of food, the presence of heavy metals and

*Author for correspondence: <Maryam.Jamshidnia58@gmail.com>. ${ }^{1}$ Department of Plant Breeding and Biotechnology, Sari Agricultural Sciences and Natural Resources University (SANRU), Sari, Iran. 2Department of Plant Breeding and Biotechnology, Gorgan University of Agricultural Sciences and Natural Resources, Gorgan, Iran. 
high acidity (Pileggi et al. 2001, Rai and Suprassana 2013, Lv et al. 2011, Nuccino et al. 1999, Serrano 1996). It is well documented to be involved in response to osmotic stresses in a wide range of organisms. Being involved in the maintenance, stability and three dimensional structures of proteins, proline also helps in maintaining the integrity of cellular structure and function (Kishore et al. 1995, Delaunay and Verma 1995, Hong et al. 2000). In plants, the first two steps of proline biosynthesis are carried out by a bi-functional enzyme known as $\Delta 1$-pyrroline-5-carboxylate synthetase (P5CS) which also plays both regulatory as well as rate limiting function in this metabolic pathway (Orcutt and Nilsen 2000, Paul and Hasegawa 1996). Verma transformed the tobacco plant by the $p 5 c s$ gene and he showed that the proline content increased in transgenic plants (Verma and Hong 2000).

The authors have introduced the cDNA of P5CS from Arabidopsis thaliana into petunia plant. Molecular and biochemical analyses were performed for the successful transformation and its efficiency in transgenic lines.

\section{Materials and Methods}

Seeds of the local petunia genotypes (Petunia violacea) were surface sterilized in $20 \%(\mathrm{v} / \mathrm{v})$ commercial bleach to which a wetting agent such as Tween 20 had been added, for ten min. The seeds were then rinsed three times with sterile water. The sterilized seeds were then germinated on MS. The procedure for optimizing regeneration using shoot apex and leaf discs was carried out according to Jamshidnia et al. 2011.

Agrobacterium strain LBA4404 harboring a plasmid pBI121 was used as the binary vector system for transformation. pBI121 contained gus gene as a reporter gene and $p 5 c s$ gene driven by the CaMV35S promoter and nptII gene driven by NOS promoter was used as a selectable marker (Fig. 1). The resulting $p 5 c s$ (originated from Arabidopsis) was used to replace the $p 5 c s$ originated from moth bean (kindly gifted by Professor Verma, Ohio University, USA) in the binary vector which included a T-DNA with two $35 \mathrm{~S}$ promoters known as pBI $p 5 c s$ (Kishore et al. 1995). P5cs from primary vector (pBluescript $p 5 c s$ ) was removed by digesting with XhoI and BamHI. This construct also carries gus gene which enables expression detection of the exogenous genes in transgenic plants. The above construct was subsequently transformed into E. coli strain DH5 $\alpha$. The binary vector containing $p 5 c s$ gene was mobilized in Agrobacterium tumefaciens strain LBA4404 using freeze and thaw method (Gelvin et al. 1989).

The Agrobacterium LBA4404 colonies containing gus gene picked up from a plate culture were incubated for $24 \mathrm{hrs}$ at $28^{\circ} \mathrm{C}$ with shaking (approximately 150 $\mathrm{rpm}$ ) in liquid LB medium (1\% tryptone, $0.5 \% \mathrm{NaCl}, 0.5 \%$ yeast extract, $\mathrm{pH} 7$, 
supplemented with $50 \mathrm{mg} /$ rifampicin and $100 \mathrm{mg} /$ kanamycin). In order to prepare bacteria for shoot tip transformation when the bacteria had reached the $\log$ phase (optical density of $0.7-0.8$ at $600 \mathrm{~nm}$ ), $5 \mathrm{ml}$ of the culture was centrifuged at $537 \times \mathrm{g}$ for 10 minutes at room temperature. The pellet was resuspended in $5 \mathrm{ml}$ fresh, antibiotic-free and modified MS liquid medium composed of MS salts and B5 vitamins supplemented with $2 \%$ sucrose, and $\mathrm{pH}$ 6.2. A $1 / 1(\mathrm{v} / \mathrm{N})$ dilution was done. In order to prepare bacteria for leaf disc transformation when the bacteria had reached the log phase (optical density of 1 at $600 \mathrm{~nm}$ ); $5 \mathrm{ml}$ of the culture was centrifuged at $537 \times \mathrm{g}$ for 10 minutes at room temperature. The pellet was resuspended in $5 \mathrm{ml}$ fresh, antibiotic-free MS liquid medium as described above, and then was diluted $1 / 20(\mathrm{v} / \mathrm{N})$ with antibiotic-free MS liquid medium.

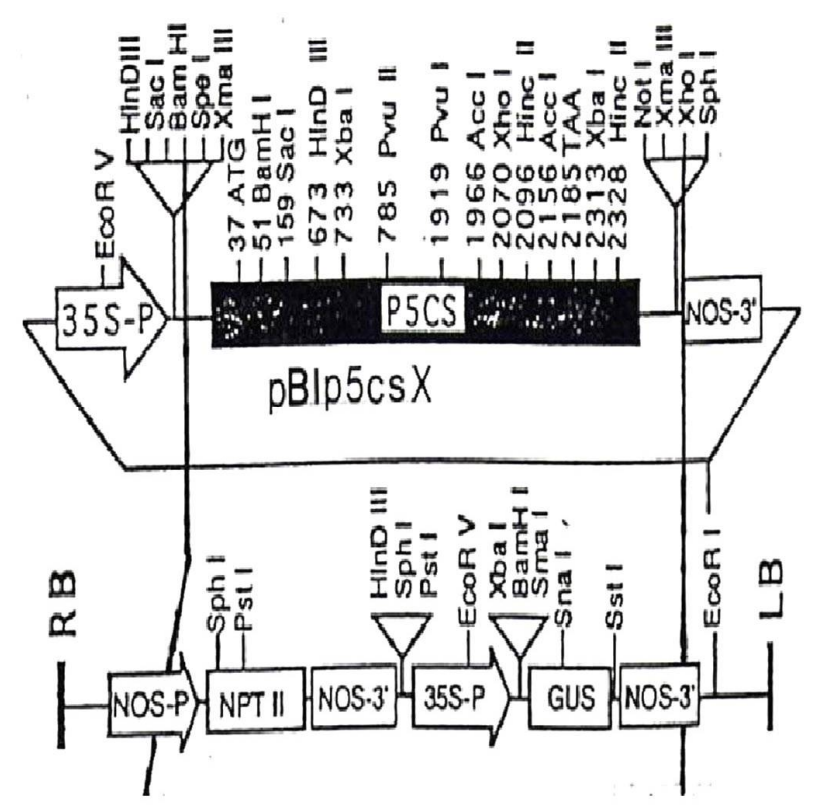

Fig. 1. The construct pBI P5CS used in transformation of petunias.

The in vitro excised shoot apices were cultured on shoot-inducing medium containing MS salts, B5 vitamins, 3\% sucrose, $0.2 \%$ Gelrite supplemented with 1 $\mathrm{mg} / \mathrm{BAP}$, adjusted to $\mathrm{pH} 5.7$ with $\mathrm{NaOH}$. After two days of culturing, isolated shoot apices were inoculated with a $5 \mu \mathrm{l}$ of $A$. tumefaciens suspension in liquid MS. The culture plate with the shoot apices and without any antibiotics was incubated for two days at $25^{\circ} \mathrm{C}$, with a light to dark period of $16 / 8 \mathrm{hrs}$. Following inoculation and incubation, the shoot apices were transferred to fresh medium and cultured for two additional days. The apices were then subcultured onto a 
selective medium (shooting medium with $500 \mathrm{mg} /$ cefotaxime and $200 \mathrm{mg} /$ kanamycin). Five replicates were taken for each treatment and the percentage of transformed plantlets were determined.

After three weeks of incubation, all explants had grown. Untransformed tissues exhibited bleaching in the presence of $200 \mathrm{mg} /$ kanamycin medium. The bleached leaves were then removed and the green tissues were transferred to the rooting medium containing MS salts, $\mathrm{B} 5$ vitamins, $3 \%$ sucrose, $0.2 \%$ gelrite supplemented with $0.1 \mathrm{mg} / \mathrm{NAA}, 500 \mathrm{mg} /$ cefotaxime and $100 \mathrm{mg} /$ kanamycin, adjusted to $\mathrm{pH} 5.7$ with $\mathrm{NaOH}$. Because shoots did not root in this condition, and died, their shoots were transferred to elongation medium containing MS salts, B5 vitamins, $0.1 \mathrm{mg} / \mathrm{GA} 3,100 \mathrm{mg} /$ kanamycin, $500 \mathrm{mg} /$ cefotaxime, 3\% sucrose, $0.2 \%$ gelrite, $\mathrm{pH}$ 5.7. After 2 - 3 weeks, shoots were large enough for excision and transferred to root-inducing medium.

Agrobacterium with OD 1 was used for co-cultivation of leaf discs regenerated from leaf disc culture. Leaf explants of tissue-cultured shoots at 4 weeks of age were scratched and cultured on shoot-inducing medium composed of MS salts, B5 vitamins and $1 \mathrm{mg} /$ folic acid supplemented with $2 \mathrm{mg} / \mathrm{BAP}, 0.1 \mathrm{mg} / \mathrm{NAA}$, $1 \mathrm{mg} /$ zeatin, $2 \%$ sucrose, $1 \%$ glucose and $0.7 \%$ agar ( $\mathrm{MS}_{3}$ medium). After three days of culturing, explants were submerged into the bacterial suspension for 5 minutes, after which they were blotted dry. The explants were plated onto the shoot-inducing medium. After 3 days of co-cultivation on $\mathrm{MS}_{3}$, the explants were washed 5 minutes with modified MS liquid medium as described above, containing $500 \mathrm{mg} /$ cefotaxime and $50 \mathrm{mg} /$ kanamycin. Explants were thereafter blotted and cultured on selective shooting medium ( $\mathrm{MS}_{3}$ containing $500 \mathrm{mg} /$ cefotaxime and $50 \mathrm{mg} /$ kanamycin) and subcultured every 2 weeks. Three replicates were taken for each treatment and the percentage of transformed plantlets were determined. After 4 weeks, green shoots were excised and were placed on root-inducing medium containing MS salts, B5 vitamins and $1 \mathrm{mg} A$ folic acid containing $2 \%$ sucrose, $1 \%$ glucose, $0.1 \mathrm{mg} / \mathrm{NAA}, 500 \mathrm{mg} \Lambda$ cefotaxime and $0.7 \%$ agar. Plants were transferred into pots when roots branched and strong enough to survive. Plants were subsequently exposed to cold stress and the leaves of such stressed plants were used for subsequent assessment of stress response. Non-transformed petunia used as controls and transformed petunia plants were conducted under continuous illumination at $4^{\circ} \mathrm{C}$. The time at which the plants were submitted to sets of temperature was 3 days. Proline assay was used for the assessment of resistance.

Total genomic DNA was extracted from green leaves of transformed and wild-type plants using the CTAB method (Murray and Thompson 1980). Molecular analysis of regenerated and transformed petunia plants was tested 
using a PCR method by gene specific primers. Forward primer was designed from the CaMV35S promoter and the reverse primer from the internal region of $p 5 c s$ gene. The sequences of the primers were 5 ' GCA CAA TCC CAC TAT CCT TCT $3^{\prime}$ as forward and 5' GAA TAA CCC TCA AAT CAA GCA $3^{\prime}$ as reverse primers. The total $25 \mu \mathrm{l} \mathrm{PCR}$ mixture contained: $1 \mu \mathrm{l}$ DNA (25-50 ng), $10 \mathrm{pmol}$ of each primer, $200 \mu \mathrm{M}$ each dNTP, $2 \mathrm{mM} \mathrm{MgCl} 2,1 x$ PCR buffer and $1 \mathrm{U}$ Taq DNA polymerase.

GUS assay was performed with control and transgenic plants (Gallagher 1992). Leaf discs from transformed and wild-type plants were prepared, and completely immersed in X-gluc solution ( $0.1 \mathrm{M}$ sodium phosphate buffer, $\mathrm{pH} 7.0$; $10 \mathrm{mM}$ EDTA; $0.5 \mathrm{mM}$ potassium ferricyanide, $\mathrm{pH} 7.0 ; 0.5 \mathrm{mM}$ potassium ferrocyanide, $\mathrm{pH} 7.0 ; 1.0 \mathrm{mM}$ X-glucuronide, and 1\% triton X100). Incubation was carried out at $37^{\circ} \mathrm{C}$ for at least $10 \mathrm{~min}$ or longer until complete bluish-green color was formed. Chlorophyll was removed using 70\% ethanol. The percentage of gus positive plantlets was determined.

One hundred mg of fresh plant material were powdered in liquid nitrogen. Ten $\mathrm{ml}$ of $3 \%$ sulfosalicylic acid were added to each sample and centrifuged at $13000 \mathrm{rpm}$ for $10 \mathrm{~min}$. Two $\mathrm{ml}$ of the supernatant from each sample and $2 \mathrm{ml}$ of acidified ninhydrin solution (1.25 g ninhydrin dissolved in $30 \mathrm{ml}$ acetic acid at boiling temperature to which $20 \mathrm{ml}$ of orthophosphoric acid were added) and 2 $\mathrm{ml}$ of $100 \%$ acetic acid were mixed and boiled for one hour. To stop further reaction, samples were immediately transferred onto ice-water and left to cool down for at least $20 \mathrm{~min}$. To each sample $4 \mathrm{ml}$ of toluene at room temperature were added, mixed thoroughly and the absorbance was measured at $520 \mathrm{~nm}$ (Bates 1973). To assay the proline content of the leaves a standard curve was prepared in the range of 0.25 to $160 \mu \mathrm{M}$ proline.

\section{Results and Discussion}

Shoot apex and leaf discs were used in order to study the regeneration and genetic transformation system of petunia. The interested construct was transformed into Agrobacterium and used for transformation of leaf discs and shoot apices. Transformed lines were selected on MS containing kanamycin as a selection marker. The results of transformation using shoot apices, showed that less time is needed for regeneration (approximately 4 weeks). The percentage of transgenic plantlets produced from shoot apices was approximately $20 \%$ higher in comparison to the leaf explants.

One mg $/$ BAP contributes to the regeneration of a large number of direct branches and multiple shoots induction, which is consistent with the results of research conducted with the foreign petunia cultivars and that the regeneration 
through the shoot apex is not related to the genotype (Jamshidnia et al. 2011). Following the regeneration of plantlets, rooted shoots were transferred to soil for them to develop into mature plants (Fig. 2A,D). The resulting putative transgenic plants were analyzed by different molecular and biochemical approaches such as genomic PCR, proline content assessment and histochemical GUS assay.
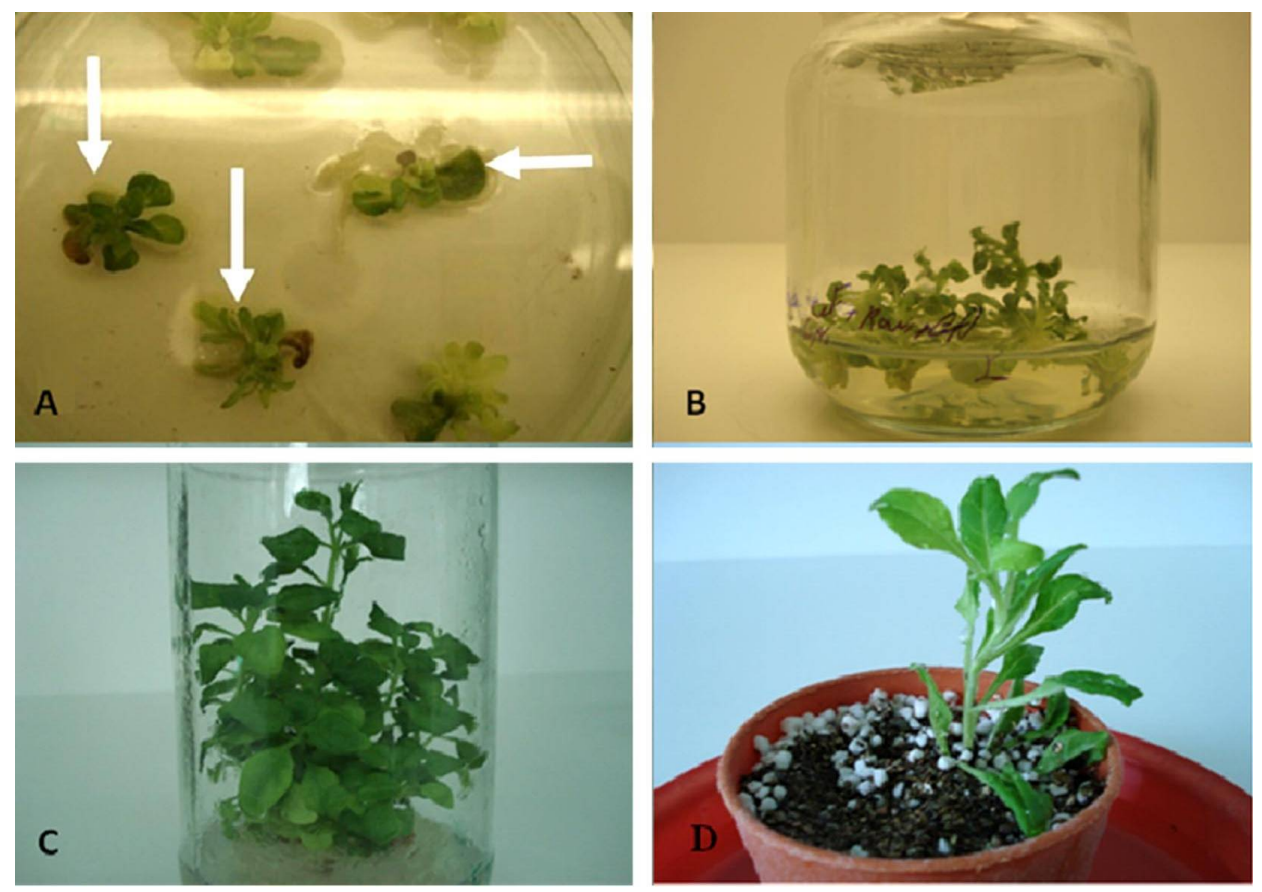

Fig. 2. Plant regeneration and genetic transformation in Iranian species of petunia. (A) Selection of transformed tissue on shoot-inducing medium containing $50 \mathrm{mg} /$ kanamycin (arrow indicates the green shoot). (B) Multiple shoots regeneration on same medium. (C) Rooting on hormone free MS. (D) Transformed plant survived on soil.

The results of histochemical assay of gus gene of transgenic plants showed high transgenic ratio in regenerated plants from shoot apex compared to leaf discs. PCR analysis confirmed the existence of gus and p5cs gene in all transformants (Figs 3 and 4). Michalczuk and Wawrzynczak (2004) used leaf explants for transformation and the highest transformation frequency $(6.15 \%)$ was reported by them, while the transformation frequency using shoot apices was approximately $70 \%$.

The plants positive for molecular and biochemical evaluations, were subsequently subjected to osmotic stress. The applied stress was applied for 
three days at $4^{\circ} \mathrm{C}$ cold stress and the response of the plants was tested by assessing the level of the produced proline in the leaf extract. In general, it is

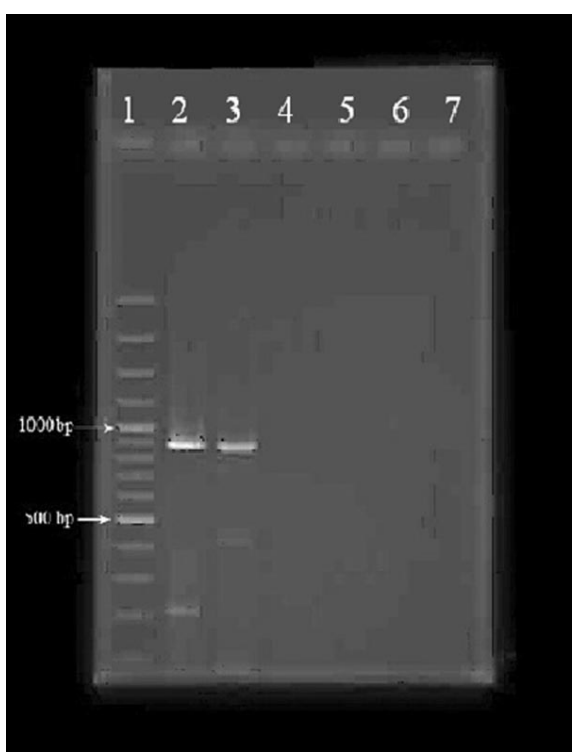

Fig. 3. Products of PCR with gus specific primers 1- Ladder 100, 2- positive control (reaction with pBI121-gus-intron plasmid), 3- reaction with genomic DNA isolated from transgenic plant, 4-6- untransformed containing plant.

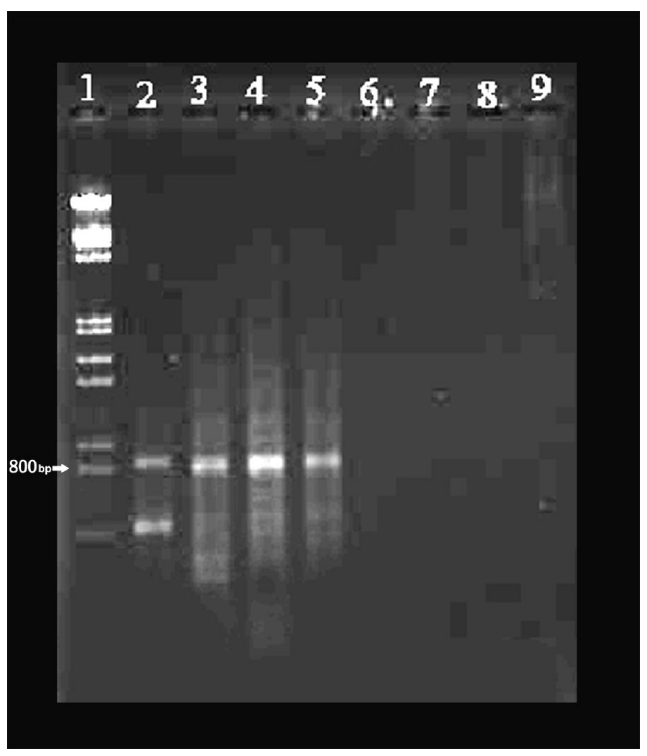

Fig. 4. PCR analysis of DNA isolated from the regenerated petunia p5cs plants: 1- marker III, 2- positive control pBI121, 3-5- reaction with genomic DNA isolated from transgenic plants, 6-8- untransformed containing plant. 
high expected that transgenic lines show higher proline production under nonstress and stress conditions due to the presence of $35 \mathrm{~S}$ viral promoter. As shown in Table 1, while both control and transgenic plants have responded to the stress by producing proline, P5CS transformed lines showed much higher proline content. $P$. violacea transformed plants contained 294 and 674 microgram per gram of fresh weight of leaf proline content in nonstress and stress condition compared with untransformed plants contained 134 and $229 \mu \mathrm{g} / \mathrm{g}$ of fresh weight of leaf in nonstress and stress condition, respectively (Table 1). Proline content of $P$. violacea transformed plants showed a significant difference between transformed plants in nonstress and stress conditions and $P$. violacea transformed plants produced the highest amount of proline content under these conditions (Fig. 5). Additionally, proline content of $P$. violacea wild type plants was significantly different compared with that observed in the transformed plants in non-stress and stress conditions.

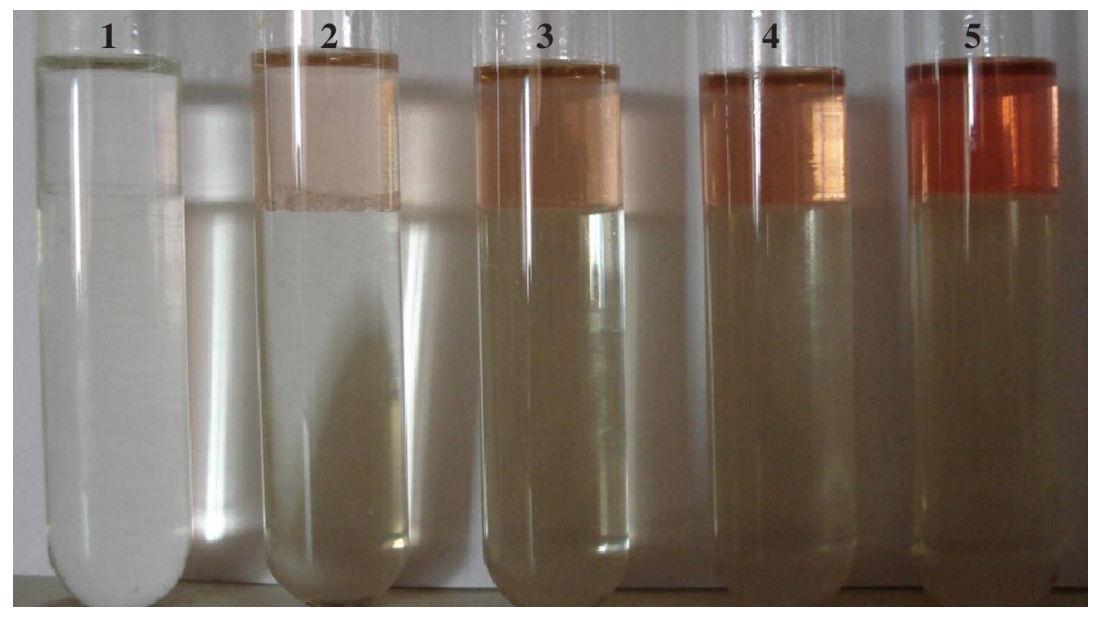

Fig. 5. Comparison of proline content in Iranian non-transgenic and transgenic petunia plants under non-stress and stress conditions 1. Blank. 2. Non-transgenic plant under non stress condition 3. Non-transgenic plant after 3 days of cold stress. 4 . Transgenic plant in non-stress conditions. 5. Transgenic plant after 3 days of cold stress.

Table 1. Proline content of fresh leaves from control and P5CS transgenic to plants grown under normal and after three days of cold stress conditions.

\begin{tabular}{lcc}
\hline Plant & $\begin{array}{c}\text { Proline content }(\mu \mathrm{g} / \mathrm{g}) \\
\text { under non stress condition }\end{array}$ & $\begin{array}{c}\text { Proline content }(\mu \mathrm{g} / \mathrm{g}) \text { after } \\
\text { three days cold condition }\end{array}$ \\
\hline Non-transformed plant & 134.14 & 229.15 \\
Transformed plant & 294.82 & 674.87 \\
\hline
\end{tabular}

After 3 days of cold stress, the increase in proline accumulation in control plants was nonsignificant (95.01 $\mu \mathrm{g} / \mathrm{g}$ of leaves). However, in the transformed 
plant, accumulation of proline could be observed which ranged from 294.82 to $674.87 \mu \mathrm{g} / g$ fresh leaves in non-stress and stress condition, respectively (Table 1). Consequently, plants with high proline content and relatively higher resistance to the stress were selected for seed formation.

There was more proline production in transformed plants $(294.82 \mu \mathrm{g} / \mathrm{g}$ of leaves) as compared to non-transgenic plants (134.14 $\mu \mathrm{g} / \mathrm{g}$ of leaves) under nonstress condition. This result is in agreement with previous reports (Kishore et al. 1995) and could be attributed to the basal activity of the strong $35 S$ promoter. On the other hand this observation further proves successful cloning of $p 5 c s$ gene and transformation of the experimental plant. It has been suggested that increasing the proline content of plants is an important physiological response to the osmotic stresses (Zonglie et al. 2000).

After 3 days of cold stress, the increase in proline accumulation in control plants was not significant but proline content was higher in transformed plants under normal and stress conditions at $4^{\circ} \mathrm{C}$ after 3 days under cold stress. This result is in agreement with those reported by Pileggi et al. (2001) who found that p5cs caused cold resistance in lettuce. However, in transformed plant, significant accumulation of proline could be observed and there was almost 2 times increase in the proline content.

In conclusion, we were able to overexpress Arabidopsis P5CS cDNA constructs into the experimental petunia plants. We have shown that plants derived through transformation show much higher proline content than non-transgenic plants. We were also able to obtain seeds from such transgenic plants. Further evaluation of these plants for their ability to tolerate osmotic stresses is in progress.

\section{References}

Bates LS, Waldren RP and Teare ID (1973) Rapid determination of free proline for water stress studies. Plant Soil. 39(1): 205-207.

Delaunay AJ and Verma DPS (1993) Proline biosynthesis and osmoregulation in plant. Plant J. 4: 215-223.

Gallagher S (1992) GUS protocol: Using the gus gene as a reporter of gene expression. (California, U.S.A.: Academic Press, NY). pp. 221.

Gelvin SB, Schilperoort RA and Verma DPS (1989) Plant Molecular Biology Manual, Supplement 1, Kluwer Academic Publishers, Dordrecht, The Netherlands.

Hong Z, Lakkineni K, Zhang Z and Verma DPS (2000) Removal of feedback inhibition of $\Delta 1$-pyrroline-5-carboxylate synthetase results in increased proline accumulation and protection of plants from osmotic stress . Plant Physiol. 122(4): 1129.

Jamshidnia M, Ghobadi C, Sayed Tabatabaei BE and Yamchi A (2011) Direct regeneration from leaf disc and shoot apex cultures of petunia. IJHST 12(3): 279-292. 
Kishore P, Hong Z, Miao GH, Hu C and Verma D (1995) Over-expression of [delta]pyrroline-5-carboxylate synthetase increases proline production and confers osmotolerance in transgenic plants. Plant Physiol. 108(4): 1387-1394.

Lv W, Lin B, Zhang M and Hua X (2011) Proline accumulation is inhibitory to Arabidopsis seedlings during heat stress. Plant Physiol. 156(4): 1921-1933.

Michalczuk B and Wawrzynczak D (2004) Effect of medium composition and date of explant drawing on effectiveness of Agrobacterium-mediated transformation in the petunia (Petunia hybrida pendula ). J. Fruit Ornam. Plant Res. 12: 5-16.

Murray MG and Thompson WF (1980) Rapid isolation of high molecular weight plant DNA. Nucleic Acid Res. 8(19): 4321-4325.

Nuccino ML, Rhodes D, McNeil SD and Hanson A (1999) Metabolic engineering of plants for osmotic stress resistance. Curr. Opinion Plant Biol. 2: 128-134.

Orcutt DM and Nilsen ET (2000) The Physiology of Plants under Stress : Soil and Biotic Factors', John Wiley, New York. pp. 684.

Paul M and Hasegawa A (1996) Annual Rev. Plant Physiol. Plant Mol. Biol. 51: 463.

Pileggi M, Pereira AAM, Silva JS, Pileggi SAV and Verma DPS (2001) An improved method for transformation of lettuce by Agrobacterium tumefaciens with a gene that confers freezing resistance. Braz. Arch. Biol. Technol. 44(2): 1-11.

Rai AN and Suprasanna P (2013) Molecular evolution of plant P5CS gene involved in proline biosynthesis. Mol. Biol. Rep. 40(11): 6429-6435.

Serrano R (1996) Salt tolerance in plants and microorganisms: toxicity targets and defence responses. Intl. Rev. Cytol. 165: 1- 52.

Verma DPS and Hong Z (2000) Removal of feedback inhibition of delta-1-pyrroline-5carboxylate synthetase results in increased proline accumulation and protection of plants from osmotic stress. Plant Physiol. 122: 1129-1136.

Zonglie H, Karuna L, Zhongming Z and Verma DPS (2000) Removal of Feedback inhibition of $\Delta$ 1-pyrroline-5-carboxylate synthetase results in increased proline accumulation and protection of plants from osmotic stress. Plant Physiol. 122: 11291136. 